\title{
Energetic deposition of metal ions: Observation of self-sputtering and limited sticking for off-normal angles of incidence
}

\author{
Hongchen $\mathrm{Wu}^{1,2}$ and André Anders ${ }^{2 *}$ \\ ${ }^{1}$ Beijing Aeronautical Manufacturing Technology Research Institute, P.O. Box 863 Beijing \\ 100024, P. R. China \\ ${ }^{2}$ Lawrence Berkeley National Laboratory, University of California, 1 Cyclotron Road, \\ Berkeley, California 94720, USA
}

Sept. 22, 2009

\section{ACKNOWLEDGMENT}

The work was supported by the China Scholarship Committee and by the US Department of Energy under Contract No DE-AC02-05CH11231 with the Lawrence Berkeley National Laboratory.

\section{DISCLAIMER}

This document was prepared as an account of work sponsored in part by the United States Government. While this document is believed to contain correct information, neither the United States Government nor any agency thereof, nor The Regents of the University of California, nor any of their employees, makes any warranty, express or implied, or assumes any legal responsibility for the accuracy, completeness, or usefulness of any information, apparatus, product, or process disclosed, or represents that its use would not infringe privately owned rights. Reference herein to any specific commercial product, process, or service by its trade name, trademark, manufacturer, or otherwise, does not necessarily constitute or imply its endorsement, recommendation, or favoring by the United States Government or any agency thereof, or The Regents of the University of California. The views and opinions of authors expressed herein do not necessarily state or reflect those of the United States Government or any agency thereof or The Regents of the University of California.

\footnotetext{
*Author to whom correspondence should be addressed. Electronic mail: aanders@lbl.gov
} 


\title{
Energetic deposition of metal ions: Observation of self-sputtering and
}

\section{limited sticking for off-normal angles of incidence}

Hongchen $\mathrm{Wu}^{1,2}$ and André Anders ${ }^{2 *}$

${ }^{1}$ Beijing Aeronautical Manufacturing Technology Research Institute, P.O. Box 863 Beijing 100024, P. R. China

${ }^{2}$ Lawrence Berkeley National Laboratory, University of California, 1 Cyclotron Road, Berkeley, California 94720, USA

\begin{abstract}
The deposition of films under normal and off-normal angle of incidence has been investigated in order to explore the relevance of non-sticking of, and self-sputtering by, energetic ions; both effects leading to the formation of neutral atoms going back to the plasma. The flow of energetic ions was obtained using a filtered cathodic arc system in high vacuum and therefore the ion flux had a broad energy distribution of typically 50-100 eV per ion. The range of materials included $\mathrm{Cu}, \mathrm{Ag}, \mathrm{Au}, \mathrm{Ti}$, and $\mathrm{Ni}$. Consistent with molecular dynamics simulations published in the literature, the experiments show that the combined effects of non-sticking and self-sputtering appear to be significant, although the relatively large error range of the method would not allow us to derive quantitative data. It was shown that modest heating and intentional introduction of oxygen background affect the results.
\end{abstract}

\footnotetext{
*Author to whom correspondence should be addressed. Electronic mail: aanders@lbl.gov
} 


\section{Introduction}

In ionized physical vapor deposition (i-PVD) processes such as pulsed laser deposition, cathodic arc deposition, and high power impulse magnetron sputtering, the ions arrive on the substrate surface not necessarily under normal incidence. A good portion of the condensable material arrives on walls, anodes, shields, shutters, baffles, etc., and here the angle of incidence may substantially depart from normal. This is especially true when three-dimensional substrates are processed under conditions where the sheath thickness is large compared to the feature size, like in via and trench filling of semiconductor microprocessors and memory devices ${ }^{[1-3]}$, especially when using plasma immersion technology ${ }^{[4-7]}$. Ions may have a relatively high probability not to stay with the surface. A non-sticking ion is very likely to be neutralized upon interaction with the surface and return to the processing volume after also having lost a portion of its kinetic energy. This has great implications for a number of process parameters and plasma properties, as well as for the resulting coating obtained on the substrate and other surfaces.

The interaction of ions with surfaces has been studied to a great extent (e.g. ${ }^{[8-12]}$ ), and the processes greatly depend on the kinetic and potential energies of the approaching ion, the surface conditions, the surface temperature, and the angle of incidence. If the ion's kinetic energy surpasses the displacement energy, which is typically $20-30 \mathrm{eV}^{[13]}$, and the approach is not too far from normal, the ion will penetrate the region below the surface and insert itself, a process known as subplantation ${ }^{[14]}$. For somewhat higher kinetic energies, collision cascades form, causing a host of processes, including sputtering ${ }^{[15-16]}$, defect formation, densification, build-up of compressive stress, and in some systems a change in the preferred orientation, which is associated with a minimization of the total free energy, which includes surface and strain energies ${ }^{[17-19]}$. At even higher ion energies (several $100 \mathrm{eV}$ and greater), compressive stress can be relieved by atomic scale annealing and relaxation of the compressed subsurface region by expansion towards the surface ${ }^{[20]}$.

Sticking probability and self-sputtering yield will affect the deposition rate, texture, and the morphology of a growing film. The sticking probability is defined as the probability that the incident ion remains bound to the solid, and the self-sputtering yield is defined as the average number of surface atoms ejected per incident ion. The sputtering yield is generally well approximated by the empirical Yamamura formula ${ }^{[21]}$, and large data collections exist for normal incident sputtering ${ }^{[15]}$. However, despite the theoretical and practical importance of the sticking probability and self-sputtering, there is only a limited number of experimental studies available for the energy range of about $20-200 \mathrm{eV}$, which is typical for cathodic arc plasmas without bias ${ }^{[22]}$. Other techniques like high power impulse magnetron sputtering (HIPIMS) ${ }^{[23-25]}$ can operate with similar energies when substrate bias is involved, although one should recognize that bias not only changes the kinetic energy but also reduces the incident angle (we always consider the angle with respect to the surface normal).

Molecular dynamics (MD) ${ }^{[26-27]}$, binary collision theory (BCT) ${ }^{[13,28]}$, and kinetic Monte-Carlo-molecular dynamics (KMC-MD) calculations ${ }^{[29]}$ have been used to model the energetic interaction of hyperthermal atoms and ions with solids ${ }^{[12]}$. This is critical for the three-dimensional metallization of semiconductor processors ${ }^{[1-2,30]}$ and oblique or glancing angle deposition ${ }^{[31-32]}$. For example, assuming perfect conditions in ultrahigh vacuum, MD models have been developed to calculate the self-sputtering yields and sticking coefficient for 
$E_{i} \geq 10 \mathrm{eV}$ ions of copper ${ }^{[12,26]}$, including for grazing incidence ${ }^{[33]}$, and for aluminum and nickel. In those studies, the surface was assumed to be either a perfect (111) structure or to be amorphous with non-zero roughness ${ }^{[27]}$. Figures 1 and 2 show examples that are typical for the results of MD calculations. They have helped explaining some observations for the energetic deposition of cathodic arc gold and zirconium plasmas on aluminum ${ }^{[34]}$ and the reduction of ion charges states in the plasma ${ }^{[35]}$.

In this contribution, experimental data are provided related to the angle-dependent sticking probability and self-sputtering. A number of different materials $(\mathrm{Cu}, \mathrm{Ag}, \mathrm{Pt}, \mathrm{Au}, \mathrm{Ti}$, and $\mathrm{Ni}$ ) have been selected for deposition experiments using streaming vacuum arc plasmas. The kinetic energy of vacuum arc plasmas, related to the supersonic ion flow from cathode spots, is material dependent but typically in the range 50-100 $\mathrm{eV}^{[22,36-38]}$. We will also show that a small addition of oxygen gas and a modest change of surface temperature can have a clear influence on the results.

\section{Experimental approach and setup}

Streaming metal plasma is produced by a pulsed filtered arc that has been described previously, e.g. ${ }^{[39-40]}$. In essence, a miniature cathodic arc plasma source with a rod cathode of $6.25 \mathrm{~mm}$ (1/4 inch) diameter is placed in the center of an annular anode body, which is grounded. The cathode is encloses by a slightly metalized alumina ceramic tubing such that the arc spots can only operate on the rod's front surface. The metallization of the ceramic is needed to enable reliable triggering of arc pulses when a sufficiently high voltage ( $>500 \mathrm{~V})$ is applied to the cathode ${ }^{[41]}$. A current flows through the metal coating of the ceramic and leads to heating and the formation of cathode spots.

The arc-initiating voltage is the charging voltage of a 10-stage pulse forming network (PFN) ${ }^{[42]}$. The PFN provides the power for 800 A pulses of $1 \mathrm{~ms}$ duration at a pulse repetition rate of 3 pulses per second. The number of pulses per deposition run was 3200 . The cathode material can be changed, and the following elements have been used in this study: $\mathrm{Cu}, \mathrm{Ag}, \mathrm{Au}, \mathrm{Ti}$, and Ni. The plasma from the arc source flows through a $90^{\circ}$-bent open filter ${ }^{[43]}$ to remove macroparticles, and the filtered plasma expanded while streaming in vacuum to the substrates placed in $16 \mathrm{~cm}$ distance from the filter exit (Fig. 3).

The experiments were done using several samples or 'deposition probes' at once in order to reduce the experimental error in the deposition thickness caused by the typical density fluctuations of cathodic arc plasma,. A deposition probe ${ }^{[44-45]}$ is, for example, a piece of cut silicon wafer $\left(\sim 1 \mathrm{~cm}^{2}\right)$ that was glued onto a pre-fabricated holder giving the probe surface a well-defined angle with respect to the incoming plasma flow. Neighboring deposition probes (normal and tilted relative to the flux) are assumed to be exposed to the same plasma incoming flow, which is reasonably justified given the large distance to the filter exit. The accuracy of the angle $\alpha$ between the surface normal of a given probe and the direction of plasma flow is determined by the holder to about $\pm 2^{\circ}$, and a systematic error of about $3^{\circ}$ should be added resulting from the finite sample size in relation to the source of plasma. The 3200 pulses resulted in a typical absolute film thickness of up to $100 \mathrm{~nm}$ as measured on the sample normal to the flow. Films on tilted samples are thinner by a factor $\cos (\alpha)$ for geometrical reasons, which will be discussed later, and their error is accordingly 
larger.

The source, filter, and sample were mounted in a cryogenically pumped vacuum chamber with a base pressure of about $1 \times 10^{-4} \mathrm{~Pa}$. The experiments were done in high vacuum except in some experimental cases when oxygen was intentionally supplied via a mass flow controller at a rate of $18 \mathrm{sccm}$, and the pumping speed was reduced to obtain a chamber pressure of $0.04 \mathrm{~Pa}$.

The sample holders were at ground potential. That implies a low-voltage sheath exist between plasma and samples in which ions arc accelerated and gain energy proportional to the sheath voltage and their charge state. The energy upon impact is therefore

$$
E_{i}=E_{0}+Q e V_{\text {sheath }} \text {, }
$$

where $E_{0}$ is the "natural" ion energy gained at cathode spots, i.e. the previously cited typical $50-100 \mathrm{eV}^{[22,36-37]}$. The plasma potential is not very far from the ground potential for most of the pulse, although it swings positive and negative at the beginning and end of the pulse, respectively, by several $10 \mathrm{~V}$, due to the changing coil current in the open filter, which is electrically in series with the arc (for more information on similar open filters and filter bias and self-bias see ${ }^{[40,46-47]}$ ).

The substrates were at room temperature for most experiments, apart from insignificant process heating, and some experiments were done at intentionally elevated temperature (up $100^{\circ} \mathrm{C}$ ) by using a radiative heater behind the substrate holders (not shown in Fig. 3). The substrate temperature was measured with a K-type thermocouple clamped to the substrate holder.

In a first approximation, the angle-dependent sticking coefficient, $S(\alpha)$, could be determined by measuring the thickness of the film on the tilted deposition probe, $d_{\alpha}$, and comparing it to the thickness on the non-tilted probe, $d_{0}$, according to

$$
S(\alpha)=\frac{d_{\alpha}}{d_{0} \cos \alpha}
$$

where the thicknesses $d_{0}$ and $d_{\alpha}$ were measured with a profilometer (Dektak IIA). For each deposition run, a whole set of deposition probes was used as indicated in Fig. 3.

The relationship (2) containing the factor $\cos (\alpha)$ is obvious from Fig. 3 but it does not include the effect of film density change as the deposition is done with non-normal or even glancing angle incidence, nor does it account for angle-dependent changes in self-sputtering and likely "cross talk" of neighboring probes. By "cross talk" we mean the deposition of material on a probe not directly originating from the incoming plasma flow but from the surface of a neighboring probe. $S(\alpha)$ would represent the absolute sticking 
coefficient only if we assumed $S(0)=1$, constant or negligible self-sputtering, and negligible cross-talk. However, self-sputtering changes with angle, too, and therefore the observed changes are due to a combination of changes in self-sputtering and sticking coefficient. For the sake of simplicity, we can consider $S(\alpha)$ as an "effective" sticking coefficient that also includes a contribution of self-sputtering. As we approach grazing incidence, $\alpha \rightarrow 90^{\circ}$, the films on the tilted surface are very thin and the error of the measurements is large. It is therefore more reasonable to plot the measured ratio

$$
R(\alpha)=\frac{d_{\alpha}}{d_{0}},
$$

and consider any deviations from the geometric factor $\cos (\alpha)$ as caused by a combination of limited sticking, self-sputtering, and “cross talk”.

\section{Results}

In a first test of the experimental approach, copper plasma was used to deposit films on the set of pairs of normal and tilted substrates, all at ground potential and room temperature, and then repeat the very same experiment under nominally identical conditions in order to show the reproducibility of the measurements. The result is shown in Fig. 4, indicating that the mean features of the curve are indeed reproducible, however, the error is relatively large as indicated by the difference between the curves. For a better mapping of the minimum near $60^{\circ}$, the experiment was repeated yet again two more times using smaller steps in angle. Those two curves are also included in Fig 4.

In a next step, the experiment was repeated but with the deposition probes slightly heated using the radiative heater. The temperature of the samples very slightly increased during the deposition run, perhaps due to a combination of process heat and that the samples had not fully reached the thermal steady state, and therefore we give both the start and end temperature for each curve shown in Fig. 5.

To learn about the sticking behavior of other elements, the cathode material was changed. The corresponding results can be sorted in two groups depending whether or not a minimum was found at an incident angle of about $60^{\circ}$ (Fig. 6). In these experiments, the samples were essentially at room temperature (apart from the insignificant process heating of a few degrees $\mathrm{K})$.

The simulations cited in the introduction already indicated that the sticking coefficient depends on the surface conditions. One possibly important factor for processing under high vacuum conditions is the presence of residual gas. Introduction of intentional background gas could highlight the relevance (or irrelevance) of the gas, and oxygen would be among the most reactive gases in the mix of background gas. Therefore, the experiments with copper plasma were repeated with oxygen fed at a rate of $18 \mathrm{sccm}$ into the chamber, and the pumping speed reduced such as to obtain a controlled pressure of $0.04 \mathrm{~Pa}$. Fig. 7 shows that there is a distinct change of the ratio $R(\alpha)$, especially for large angles. 


\section{Discussion}

The filtered cathodic arc plasma is well suited for this kind of experiment because it has the advantage of representing a large flux of ions with very limited contribution of neutrals: one can argue that any neutrals produced in the cathode region are not transported through the macroparticle filter, i.e. the curved coil is both a macroparticle and neutral atom filter $\left({ }^{[40]}\right.$ and ${ }^{[48]}$ chpt. 7). Yet, the main point of the investigation is to show that the flow of fully ionized metal plasma itself is a source of neutrals when interacting with the surface of a solid. An effective sticking coefficient $S(\alpha)$ was introduced that includes the contribution of self-sputtering when ion arrives at the substrate. We must accept that the relative contributions of non-sticking and self-sputtering cannot be deconvoluted in this kind of experiment. Additionally, we acknowledge that the method does not provide absolute values for the sticking coefficient.

The cathodic arc plasma is known to be fluctuating or 'noisy', therefore it should not surprise that each repetition of the same experiment results in a slightly different result. The values of Fig. 4 indicate what "slightly different" means. One can also suspect that some "cross talk" between neighboring samples exists: the material that does not stick needs to go somewhere, and with some probability it will end up on the reference sample that is not tilted as well as on the other samples nearby. Evidence for this is the appearance of data points with $R(\alpha)>\cos (\alpha)$.

The rather large changes of the effective sticking coefficient for the small changes in temperature, as indicated by the deviation of $R(\alpha)$ from the ideal $\cos (\alpha)$ function in Fig. 5 , is somewhat surprising (as it is common in material science, the change in temperature could be normalized by the melting temperature of the material, hence the assessment that the temperature change is 'small'). One possible factor for the effect is that the heating of the samples and surrounding region is usually accompanied by a rise of the residual gas pressure from the low $10^{-4} \mathrm{~Pa}$ to the mid $10^{-3} \mathrm{~Pa}$. The residual gas changes the surface conditions, which are known to be critical for the sticking probability and self-sputtering yield. The great sensitivity to the surface conditions is also evident from the simulations shown in Fig. 1: here we see a relatively large effect, especially at large angles, even as the chemical composition is not changed but just the arrangement of atoms and the atomic-scale roughness. Chemical changes affect the surface binding energy more profoundly, and one must expect that changes of the effective sticking coefficient $S(\alpha)$, and of the measured ratio $R(\alpha)$, are at least as strong as the effects of atom rearrangement. With these arguments in mind, our interpretation "blaming" the residual gas is supported by the experiment using intentionally introduced oxygen background gas (Fig. 7).

The results of Fig. 6 show that, at least qualitatively, all materials show non-sticking and/or self-sputtering, and the effect is stronger for materials grouped in Fig. 6(a). A deviation of the curve below the projection factor $\cos (\alpha)$ indicates limited sticking and 
self-sputtering, and a deviation above the curve deposition of material originating from other surfaces ("cross talk"). From the theoretical knowledge and simulation of sticking and self-sputtering, e.g. Figs. 1 and 2, it should be expected that the curves $R(\alpha)$ are below the $\cos (\alpha)$ curve especially in the range $30^{\circ}$ to $70^{\circ}$.

Sputtered atoms are very likely to be neutral, and non-sticking ions are very likely to be neutralized above the surface by classical over-the-barrier and Auger electron emission, surface plasma excitation and other processes ${ }^{[10,49-51]}$. Thus material leaving the surface will return to the plasma volume as atoms.

This line of thought is in agreement with work by Miller who estimated that self-sputtering alone will limit the effective ion condensation coefficient of vacuum arc copper plasma to at most $0.94{ }^{[52]}$. Consequently, as argued in earlier papers ${ }^{[35,53]}$, the appearance of neutral atoms from surfaces exposed to plasma will necessarily lead to a reduction of the local degree of ionization as well as to a reduction of the mean ion charge state due to non-resonant charge exchange collisions between multiply charged ions and neutral atoms.

\section{Conclusion}

The deposition experiments described show that, as expected from computer simulations, self-sputtering and non-sticking play an important role when a flow of energetic (50-100 eV) ions interacts with tilted surfaces. The experimental results for all materials investigated $(\mathrm{Cu}, \mathrm{Ag}, \mathrm{Au}, \mathrm{Ti}$, and $\mathrm{Ni}$ ) are in qualitatively in agreement with the computational literature data. It confirms that the flow of fully ionized plasma itself is a source of neutrals when interacting with surfaces. The present experiments, however, do not allow us to separate the effects of limited sticking and self-sputtering, nor can we derive quantitative values for combined non-sticking and self-sputtering that match the precision of computer simulation. However, it was experimentally shown that the effective sticking coefficient depend in a sensitive manner on surface conditions, especially on the presence of a reactive background gas.

\section{Acknowledgments}

The work was supported by the China Scholarship Committee and by the US Department of Energy under Contract No DE-AC02-05CH11231. 


\section{References}

[1] Monteiro O R J. Vac. Sci. Technol. B 199917 1094-1097

[2] Siemroth P, Wenzel C, Kliomes W, et al. Thin Solid Films 1997308 455-459

[3] Zhong G and Hopwood J J. Vacuum Sci. Technol. B 199917 405-409

[4] Ikehata T, Shioya K, Araki T, et al. Nucl. Instrum. Meth. Phys. Res. B 2003206 772-776

[5] Kim D and Economou D J J. Vacuum Sci. Technol. B 200321 1248-1253

[6] Mändl S, Thorwart G, Huber P, et al. Surf. Coat. Technol. 2001 139 81-86

[7] Yukimura K, Ma X and Ikehata T Surf. Coat. Technol. 2004186 73-76

[8] Hagstrum H D Phys. Rev. 1956104 1516-1527

[9] Auciello O and Kelly R "Ion Bombardment Modification of Surfaces". Amsterdam: Elsevier 1984.

[10] Burgdörfer J, Lerner P and Meyer F W Phys. Rev. A 1991445674

[11] Aumayr F and Winter H Phil. Trans. R. Soc. Lond. A 2004362 77-102

[12] Coronell D G, Hanson D E, Voter A F, et al. Appl. Phys. Lett. 199873 3860-3862

[13] Eckstein W Computer Simulation of Ion-Solid Interactions. Berlin: Springer-Verlag, 1991

[14] Lifshitz Y, Kasi S R and Rabalais J W Phys. Rev. Lett. 198962 1290-1293

[15] Matsunami N, Yamamura Y, Itikawa Y, et al. Atomic Data and Nuclear Data Tables 198431 1-80

[16] Behrisch R and Eckstein W "Sputtering by Particle Bombardment". Berlin: Springer 2007.

[17] McKenzie D R and Bilek M M M Thin Solid Films 2001382 280-287

[18] Mukherjee S, Prokert F, Richter E, et al. Surf. Coat. Technol. 2004186 99-103

[19] Zhang J-M, Xu K-W and Ji V Appl. Surf. Sci. 2001180 1-5

[20] Bilek M M M and McKenzie D R Surf. Coat. Technol. 2006200 4345-4354

[21] Yamamura Y, Matsunami N and Itoh N Radiation Effects and Defects in Solids 198371 $65-86$

[22] Anders A and Yushkov G Y J. Appl. Phys. 200291 4824-4832

[23] Ehiasarian A P, Hovsepian P E, Hultman L, et al. Thin Solid Films 2004457 270-277

[24] Bohlmark J, Lattemann M, Gudmundsson J T, et al. Thin Solid Films 2006515 1522-1526

[25] Alami J, Eklund P, Andersson J M, et al. Thin Solid Films 2007515 3434-3438

[26] Kress J D, Hanson D E, Voter A F, et al. J. Vac. Sci. Technol. A 199917 2819-2825

[27] Hanson D E, Stephens B C, Saravanan C, et al. J. Vac. Sci. Technol. A 200119 820-825

[28] Hou M and Eckstein W J. Appl. Phys. 199271 3975-3980

[29] Pomeroy J M, Jacobsen J, Hill C C, et al. Phys. Rev. B 200266 235412-1-8

[30] Hopwood J Phys. Plasmas 19985 1624-1631

[31] Robbie K and Brett M J J. Vac. Sci. Technol. A 199715 1460-1465

[32] Lakhtakia A and Messier R Sculptured Thin Films: Nanoengineered Morphology and Optics. Bellingham, WA: SPIE - The International Society for Optical Engineering, 2005

[33] Hanson D E, Kress J D, Voter A F, et al. Phys. Rev. B 199960 11723-11729

[34] Anders A Appl. Phys. Lett. 200485 6137-6139 
[35] Anders A IEEE Trans. Plasma Sci. 200533 205-209

[36] Plyutto A A, Ryzhkov V N and Kapin A T Sov. Phys. JETP 196520 328-337

[37] Kutzner J and Miller H C IEEE Trans. Plasma Sci. 198917 688-694

[38] Byon E and Anders A J. Appl. Phys. 200393 1899-1906

[39] MacGill R A, Dickinson M R, Anders A, et al. Rev. Sci. Instrum. 199869 801-803

[40] Anders A Surf. Coat. Technol. 1999 120-121 319-330

[41] Anders A, Brown I G, MacGill R A, et al. J. Phys. D: Appl. Phys. 199831 584-587

[42] Anders A, MacGill R A and McVeigh T A Rev. Sci. Instrum. 199970 4532-4534

[43] Anders A J. Phys. D: Appl. Phys. 200740 2272-2284

[44] Anders A, Anders S and Brown I G Plasma Sources Sci. Technol. 19954 1-12

[45] Bilek M M M and Brown I G Rev. Sci. Instrum. 199869 3353-3356

[46] Anders A, Anders S and Brown I G J. Appl. Phys. 199475 4900-4905

[47] Anders A and MacGill R A Surf. Coat. Technol. 2000 133-134 96-100

[48] Anders A Cathodic Arcs: From Fractal Spots to Energetic Condensation. New York: Springer, 2008

[49] Hagstrum H D Phys. Rev. 195496 336-365

[50] Hagstrum H D Phys. Rev. 1966150495

[51] Lee H-W and George T F Surf. Sci. 1985159 214-232

[52] Miller H C J. Phys. D: Appl. Phys. 197912 1293-1298

[53] Anders A, Oks E M and Yushkov G Y J. Appl. Phys. 2007102043303 


\section{Figure Captions}

Fig. 1 MD simulation of the angle-dependent sticking coefficient $S(\alpha)$ of aluminum ions impacting a perfect Al (111) surface (top) and an amorphous Al surface with non-zero roughness (bottom), data from ${ }^{[27]}$.

Fig. 2 MD simulation of the angle-dependent sticking coefficient $S(\alpha)$ (top) and self-sputtering yield (bottom) of nickel ions impacting a perfect Ni (111) surface (data from $\left.{ }^{[27]}\right)$.

Fig. 3 Experimental setup of a filtered pulsed cathodic arc system depositing thin films on tilted and non-tilted substrates; one of the tilted/non-tilted substrate pairs is shown in the lower left corner of the figure.

Fig. 4 Two individual deposition runs under nominally equal conditions including the whole angle span; the similarity of the curves they indicate the reproducibility of the experiment, though relatively large errors appear for glancing angle deposition. The function $\cos (\alpha)$ is shown to show the pure geometric effect of the incident angle on the film thickness. The experiments were repeated in the angle range $45^{\circ}-70^{\circ}$ with smaller angle steps. In all cases, the samples were at ground potential and the plasma was vacuum-arc-generated copper.

Fig. 5 Ratio $R(\alpha)$ as per definition (3) as a function of incident angle $\alpha$ for slightly heated substrates.

Fig. 6 Ratio $R(\alpha)$ as per definition (3), as a function of incident angle $\alpha$, for various cathodic arc plasmas; the samples were at ground potential and at room temperature.

Fig. 7 Ratio $R(\alpha)$ as per definition (3), as a function of incident angle $\alpha$, for copper plasma in the absence (high vacuum, $10^{-4} \mathrm{~Pa}$ ) and presence of oxygen background gas. 


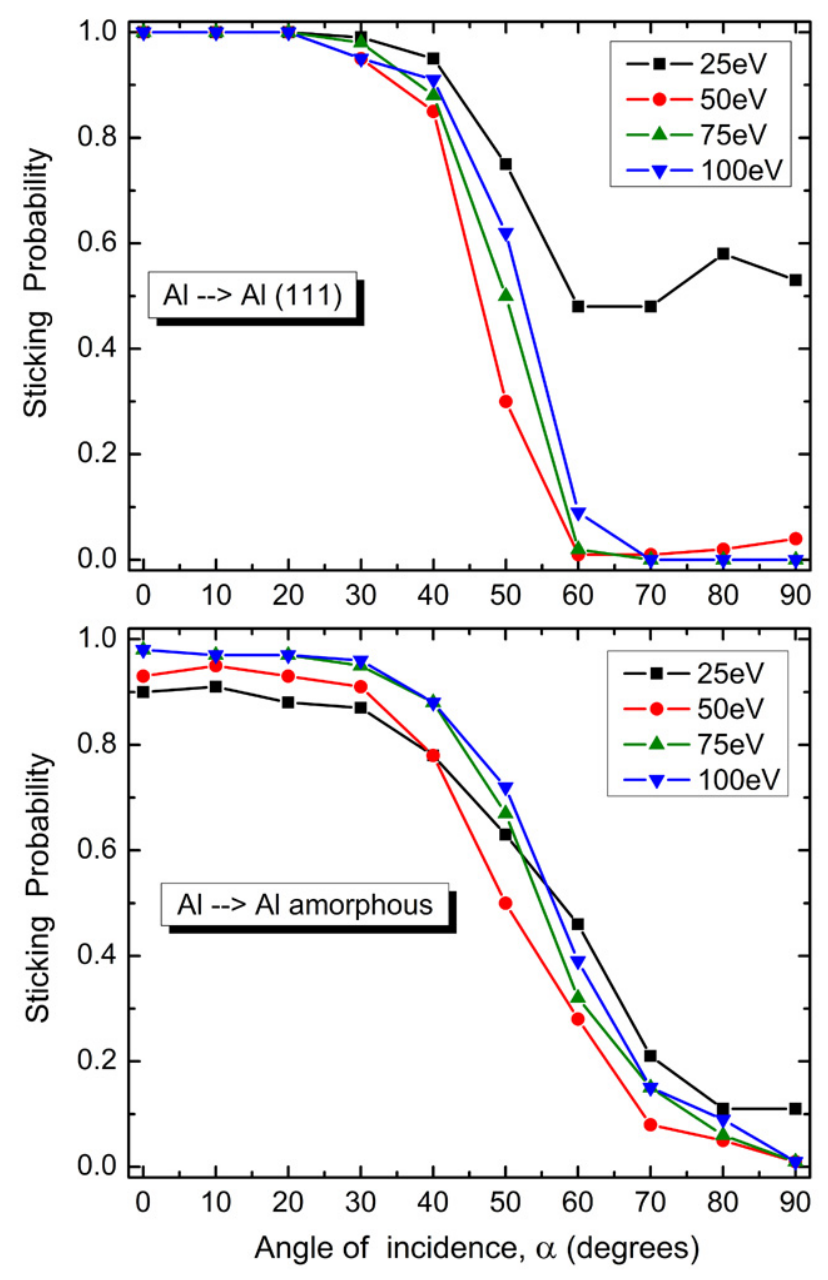

Fig. 1 

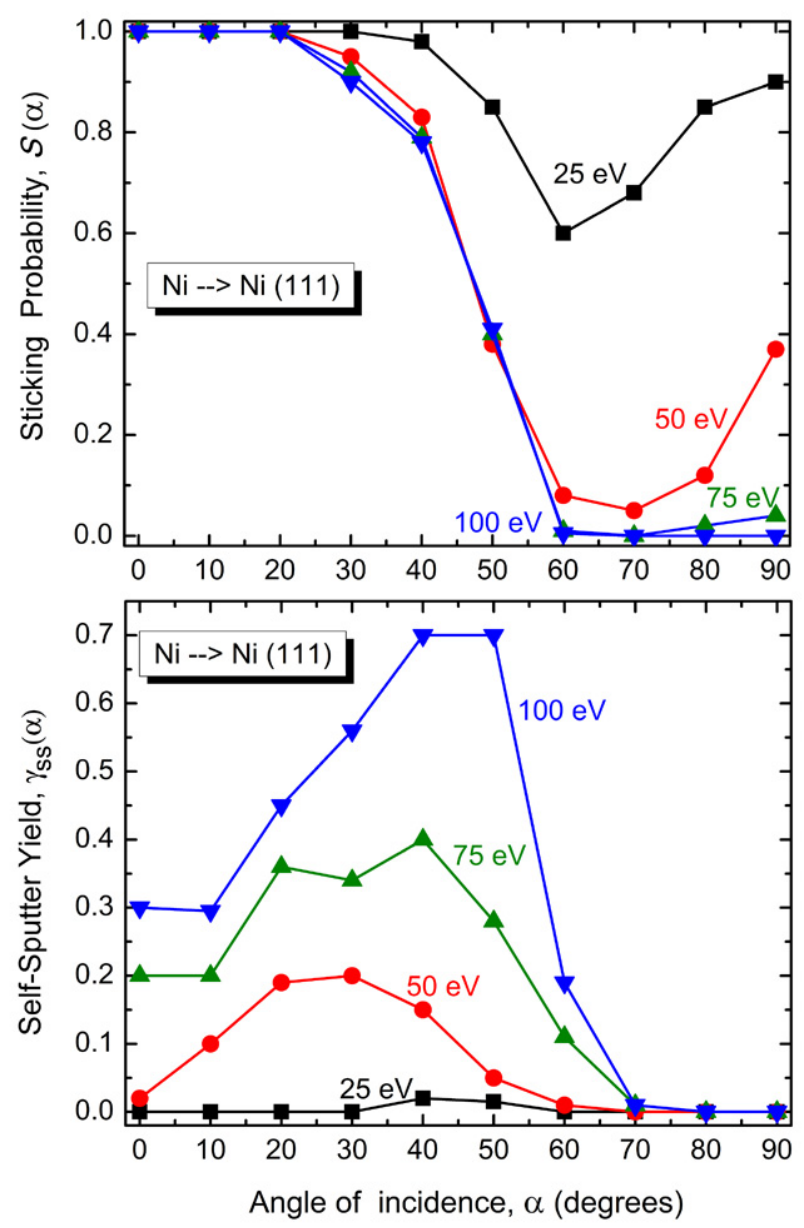

Fig. 2 


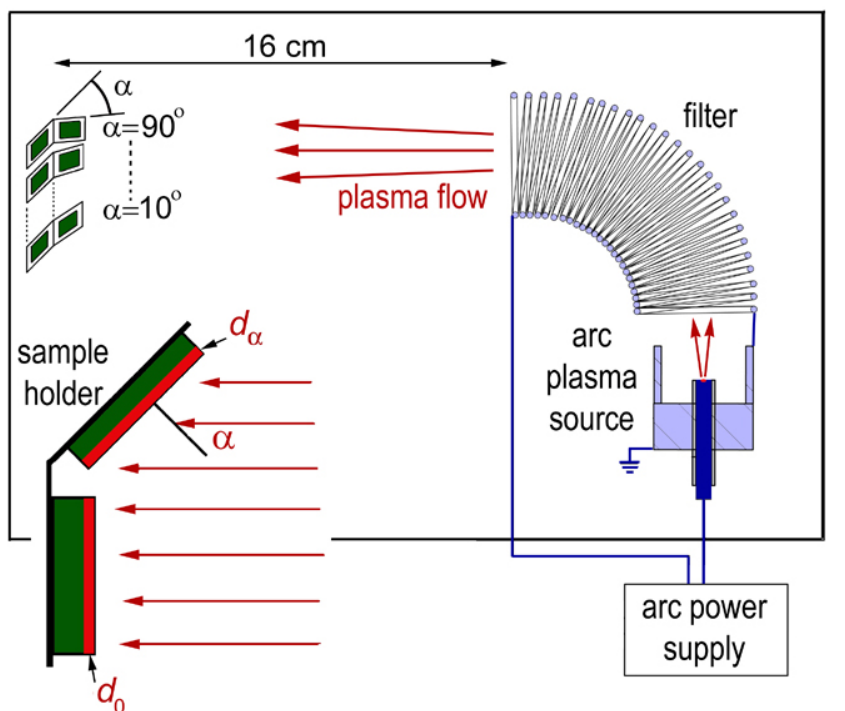

Fig. 3 


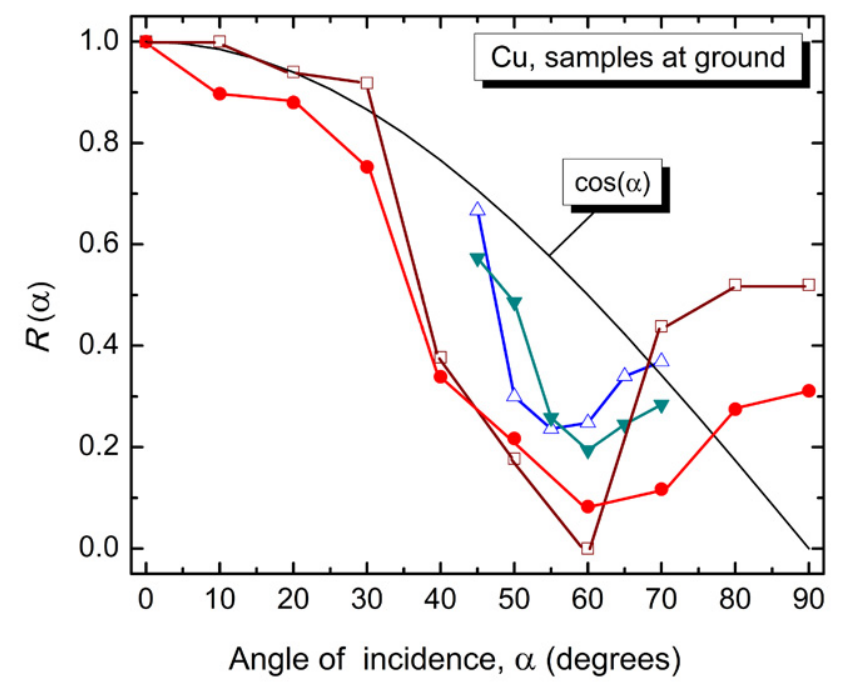

Fig. 4 


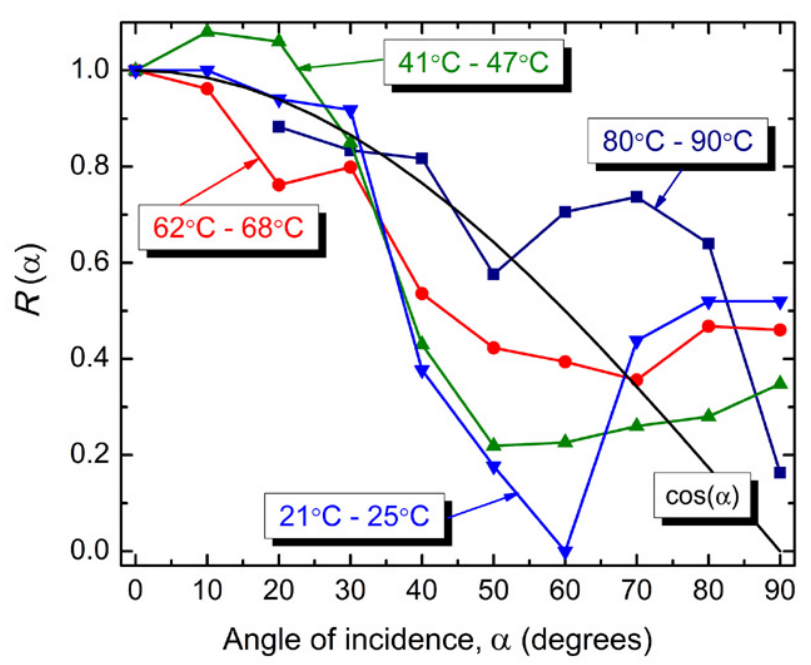

Fig. 5 

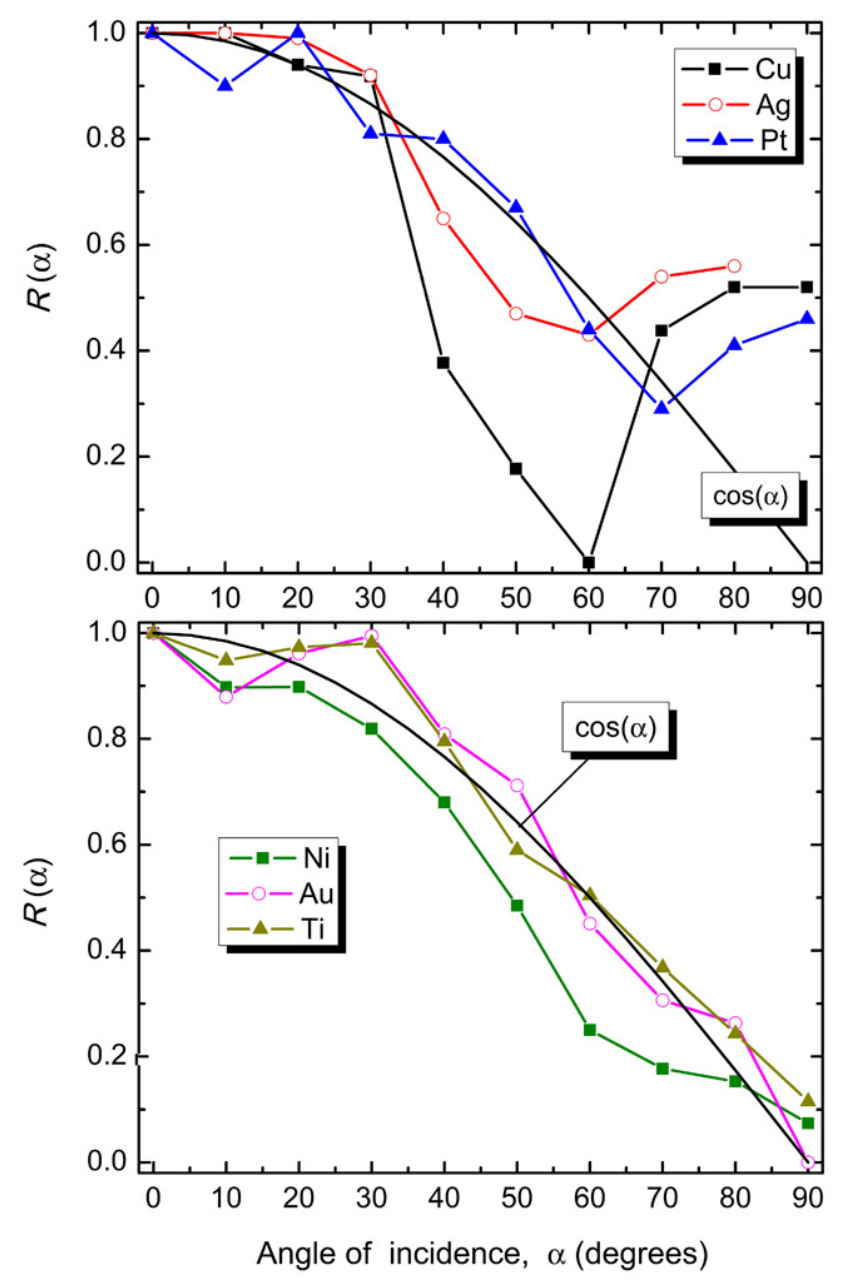

Fig. 6 


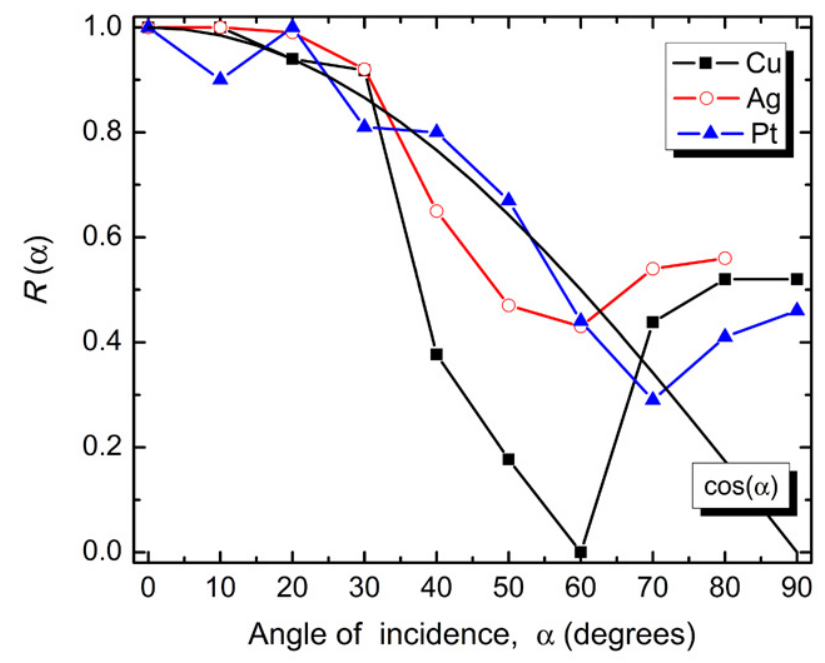

Fig. 7 